\title{
SOME ASYMPTOTIC EXPRESSIONS IN THE THEORY OF NUMBERS*
}

BY

\section{T. H. GRONWALL}

While formerly the research of asymptotic expressions in the theory of numbers was largely confined to the approximate determination of the summatory function (or the mean value) of a given arithmetic function, recent progress in the theory of prime numbers has opened a new field for asymptotic investigations, viz., the research of upper and lower limits of an arithmetic function for large values of its argument.

The first result in this line was obtained by LANDAU: $\dagger$

$$
\liminf _{x=\infty} \frac{\varphi(x)}{\frac{x}{\log \log x}}=e^{-c}, \quad \limsup _{x=\infty} \frac{\varphi(x)}{x}=1,
$$

where $\varphi(x)$ is the number of relative primes to $x$ which are $<x$, and $C$ the Eulerian constant. For the number $T(x)$ of divisors of $x$, WIGERT $\ddagger$ has further shown that

$$
\liminf _{x=\infty} T(x)=2, \quad \limsup _{x=\infty} \frac{\log T(x)}{\frac{\log x}{\log \log x}}=\log 2 .
$$

In the present paper, I propose to give a similar investigation of the function

$$
s_{a}(x)=\sum_{d 1} d^{a},
$$

the sum extending over all divisors $d$ of the integer $x$. For $\alpha=0$, $s_{a}(x)=T(x)$, and as we have

or

$$
s_{-a}(x)=\sum_{d \mid x} d^{-a}=\sum_{d d^{\prime}=x} d^{-a}=\sum_{d d^{\prime}=x} d^{\prime^{-a}}=\sum_{d d^{\prime}=x}\left(\frac{x}{d}\right)^{-a}=x^{-a} \sum_{d d^{\prime}=x} d^{a}
$$

$$
s_{-a}(x)=x^{-a} s_{a}(x),
$$

it is obviously sufficient to consider the case $\alpha>0$. As 1 and $x$ are divisors

* Presented to the Society (Chicago) April 5, 1912.

†E. LaNdad, Handbuch der Lehre von der Verteilung der Primzahlen, Leipzig, 1909, pp. 217-219. In the following, this work will be briefly quoted as "Handbuch."

$\ddagger$ Handbuch, pp. 219-222. 
of $x$, we have

$$
s_{a}(x) \overline{>} 1+x^{\alpha},
$$

the equality sign prevailing when $x$ is a prime number, and consequently

$$
\liminf _{x=\infty} \frac{s_{a}(x)}{x^{a}}=1, \quad \alpha>0
$$

To obtain the corresponding superior limit, we express $s_{a}(x)$ in terms of the prime factors of $x$. We denote the prime numbers, in their natural order, by $p_{1}(=2), p_{2}(=3), p_{3}(=5), \cdots, p_{n}, \cdots$, and decompose $x$ into prime factors:

$$
x=p_{\lambda_{1}}^{\nu_{1}} \cdot p_{\lambda_{8}}^{\nu_{2}} \cdot \cdots \cdot p_{\lambda_{n}}^{\nu_{n}}
$$

where

$$
\lambda_{1}<\lambda_{2}<\cdots<\lambda_{n} \quad \text { and } \quad \nu_{1}>0, \quad \nu_{2}>0, \cdots, \quad \nu_{n}>0 .
$$

We then obviously have

$$
s_{\alpha}(x)=\sum_{\mu_{1}=0}^{\nu_{1}} \cdots \sum_{\mu_{n}=0}^{\nu_{n}} p_{\lambda_{1}}^{a \mu_{1}} \cdots p_{\lambda_{n}}^{a \mu_{n}}=\prod_{k=1}^{n}\left(\sum_{\mu_{k}=0}^{\nu_{k}} p_{\lambda_{k}}^{a \mu_{k}}\right),
$$

whence the formula*

$$
s_{a}(x)=\prod_{k=1}^{n} \frac{p_{\lambda_{k}}^{a\left(\nu_{k}+1\right)}-1}{p_{\lambda_{k}}^{a}-1}=x^{a} \prod_{k=1}^{n} \frac{1-\frac{1}{p_{\lambda_{k}}^{a\left(\nu_{k}+1\right)}}}{1-\frac{1}{p_{\lambda_{k}}^{a}}} .
$$

We now distinguish three cases: $\alpha>1, \alpha=1$, and $0<\alpha<1$.

First case, $\alpha>1$. - This case may be treated in a quite elementary way. From (5) it follows that

$$
s_{a}(x)<x^{a} \prod_{k=1}^{n} \frac{1}{1-\frac{1}{p_{\lambda_{k}}^{a}}}<x^{a} \prod_{p} \frac{1}{1-\frac{1}{p^{a}}}
$$

where the second product extends over all prime numbers, $\uparrow$ and the wellknown relation $\ddagger$

$$
\prod_{p} \frac{1}{1-\frac{1}{p^{a}}}=\sum_{n=1}^{\infty} \frac{1}{n^{\alpha}}=\zeta(\alpha)
$$

* This formula may be found in any elementary text book on the theory of numbers.

† In fact, the second product arises from the first by multiplication by all factors $\frac{1}{1-\frac{1}{p^{a}}}$

where $p$ does not divide $x$, and each of these factors is obviously $>1$.

$\ddagger$ This formula may be found in any elementary text book on the theory of numbers. 
for $\alpha>1$, where $\zeta(\alpha)$ is the Riemann Zeta function, gives

whence

$$
s_{\alpha}(x)<x^{\alpha} \zeta(\alpha)
$$

$$
\limsup _{x=\infty} \frac{s_{a}(x)}{x^{\alpha}} \leqq \zeta(\alpha)
$$

In order to show that the equality sign prevails in (7), the obvious way is to construct a special infinite sequence of integers $x_{1}, x_{2}, \cdots x_{n}, \cdots$ such that

$$
\lim _{n=\infty} \frac{s_{a}\left(x_{n}\right)}{x_{n}^{a}}=\zeta(\alpha)
$$

To this purpose, make

we then obtain from (5)

$$
x_{n, \nu}=\left(p_{1} p_{2} \cdots p_{n}\right)^{\nu-1} \quad\left(\begin{array}{l}
n=1,2,3, \cdots \\
\nu=2,3,4, \cdots
\end{array}\right)
$$

Now the infinite product

$$
s_{a}\left(x_{n, v}\right)=x_{n, v}^{a} \prod_{k=1}^{n} \frac{1-\frac{1}{p_{k}^{v a}}}{1-\frac{1}{p_{k}^{a}}} .
$$

$$
\prod_{p} \frac{1-\frac{1}{p^{\nu a}}}{1-\frac{1}{p^{\alpha}}}=\frac{\zeta(\alpha)}{\zeta(\nu \alpha)}
$$

is uniformly convergent in respect to $\nu$ for $\nu \bar{\Sigma} 2 ;^{*}$ to any given $\epsilon>0$ we may therefore find an $n(\epsilon)$ independent of $\nu$ such that for $n \overline{\bar{\Sigma}} n(\epsilon)$ and $\nu \bar{\Sigma} 2$

Furthermore

$$
\frac{\zeta(\alpha)}{\zeta(\nu \alpha)}+\frac{\epsilon}{2}>\prod_{k=1}^{n} \frac{1-\frac{1}{p_{k}^{\nu \alpha}}}{1-\frac{1}{p_{k}^{\nu}}}>\frac{\zeta(\alpha)}{\zeta(\nu \alpha)}-\frac{\epsilon}{2}
$$

$$
\lim _{\nu=\infty} \zeta(\nu \alpha)=\lim _{\nu=\infty} \sum_{n=1}^{\infty} \frac{1}{n^{\nu \alpha}}=1
$$

* For the truth of this statement, it is necessary and sufficient that

$$
\sum_{p} \frac{1}{p^{v a}}
$$

be uniformly convergent in respect to $\nu$ for $\nu \geqq 2$. This is immediately seen to be the case, as for $\nu \geqq 2, \alpha>1$

$$
\frac{1}{p^{\nu a}}<\frac{1}{p^{2}}
$$

and $\underset{p}{\mathrm{x}} \frac{1}{p^{2}}$ is convergent, each term being also a term in the convergent series $\sum_{n=1}^{\infty} \frac{1}{n^{2}}$. 
we may therefore determine a $\nu(\epsilon)$ such that for $\nu \overline{>} \nu(\epsilon)$

$$
\zeta(\alpha)+\frac{\epsilon}{2}>\frac{\zeta(\alpha)}{\zeta(\nu \alpha)}>\zeta(\alpha)-\frac{\epsilon}{2} .
$$

From (9), (10) and (11) it then follows that

$$
x_{n, \nu}^{a}(\zeta(\alpha)+\epsilon)>s_{\alpha}\left(x_{n, \nu}\right)>x_{n, \nu}^{a}(\zeta(\alpha)-\epsilon)
$$

for $n \bar{\Sigma} n(\epsilon)$ and $\nu \bar{\nu} \nu(\epsilon)$. Now if we make $\nu=n+1$ and

$$
x_{n}=x_{n, n+1}=\left(p_{1} p_{2} \cdots p_{n}\right)^{n}
$$

it follows that for $n \overline{>}$ the larger of the numbers $n(\epsilon)$ and $\nu(\epsilon)-1$,

$$
\zeta(\alpha)+\epsilon>\frac{s_{a}\left(x_{n}\right)}{x_{n}^{\alpha}}>\zeta(\alpha)-\epsilon,
$$

that is, (8) is satisfied, and the combination of (7) and (8) then gives the desired expression

$$
\limsup _{x=\infty} \frac{s_{a}(x)}{x^{a}}=\zeta(\alpha),
$$

for $\alpha>1$.

Second case, $\alpha=1$. -This case (as well as the third one) is not accessible by the elementary method of the first case, but requires the use of some of the simplest problems in the analytical theory of prime numbers. The two principal arithmetic functions used in this theory are $\pi(x)$, denoting the number of primes $\bar{\Sigma} x$, and $\vartheta(x)$, denoting the sum of the logarithms of these primes:

$$
\pi(x)=\sum_{p \leq x} 1, \quad \vartheta(x)=\sum_{p \leqq x} \log p .
$$

The main object of the theory in question is the derivation of asymptotic formulæ for $\pi(x)$ and $\vartheta(x)$ when $x$ is large, the simplest results in this direction being

$$
\begin{gathered}
\lim _{x=\infty} \frac{\pi(x) \log x}{x}=1, \\
\lim _{x=\infty} \frac{\vartheta(x)}{x}=1 .^{*}
\end{gathered}
$$

Of the more accurate asymptotic expressions, we shall here need only the following one $\dagger$

$$
\pi(x)=\frac{x}{\log x}+O\left(\frac{x}{\log ^{2} x}\right) . \ddagger
$$

\footnotetext{
* Handbuch, p. 193.
}

$\dagger$ Handbuch, p. 196.

$\ddagger$ The symbols $O$ and $O$, which are very useful in asymptotic calculations, are defined in the following manner (Handbuch, p. 59-62): 
Returning to our problem, we define an integer $n_{x}$ by the conditions

$$
\prod_{k=1}^{n} p_{k} \bar{\sum} x<\prod_{k=1}^{n_{k}+1} p_{k}
$$

then by (13)

$$
\vartheta\left(p_{n_{s}}\right) \overline{l o g} x<\vartheta\left(p_{n_{s}+1}\right),
$$

and as it easily follows from (14) that

$$
\lim _{n=\infty} \frac{p_{n+1}}{p_{n}}=1,
$$

we conclude by the aid of (15) that

$$
\begin{aligned}
& 1=\lim _{x=\infty} \frac{\vartheta\left(p_{n_{\varepsilon}}\right)}{p_{n_{x}}} \sum \lim _{x=\infty} \frac{\log x}{p_{n_{x}}} \sum \lim _{x=\infty} \frac{\vartheta\left(p_{n_{x}+1}\right)}{p_{n_{z}}}=\lim _{x=\infty} \frac{p_{n_{x}+1}}{p_{n_{z}}} \cdot \lim _{x=\infty} \frac{\vartheta\left(p_{n_{x}+1}\right)}{p_{n_{z}+1}} \\
& \text { or } \\
& =1 \cdot 1=1 \text {, }
\end{aligned}
$$

$$
\lim _{x=\infty} \frac{\log x}{p_{n_{z}}}=1, \quad \lim _{x=\infty} \frac{p_{n_{z}}}{\log x}=1 ;
$$

when ce finally, by the definition of the symbol $o$,

$$
p_{n_{s}}=\log x \cdot(1+o(1)) \text {. }
$$

We furthermore have, $C$ being the Eulerian constant,

$$
\prod_{p \leq x}\left(1-\frac{1}{p}\right)=\frac{e^{-c}}{\log x}(1+o(1)),,^{*}
$$

The notation $f(x)=O(g(x))$ signifies that $\limsup _{x=\infty} \frac{|f(x)|}{g(x)}$ is finite, so that a positive constant $A$ may be found such that $|f(x)|<A g(x)$ for all sufficiently large values of $x$. Examples: $\sqrt{x}=O(x), x+1=O(x), 1 / x^{2}=O\left(1 / x^{3 / 2}\right), \sin x=O(1)$.

The notation $f(x)=o(g(x))$ signifies that $\lim _{x=\infty} \frac{f(x)}{g(x)}=0$.

Examples: $\log x=o(\sqrt{x}), 1 / x=o(1)$.

These definitions immediately give the following rules for calculation:

I. If $f_{1}(x)=O\left(g_{1}(x)\right)$ and $f_{2}(x)=O\left(g_{2}(x)\right)$, then $f_{1}(x)+f_{2}(x)=O\left(g_{1}(x)+g_{2}(x)\right)$ and if $f_{1}(x)=o\left(g_{1}(x)\right)$ and $f_{2}(x)=o\left(g_{2}(x)\right)$, then $f_{1}(x)+f_{2}(x)=o\left(g_{1}(x)+g_{2}(x)\right)$.

II. In a sum of several symbols $O$ or $o$, only the one of the highest order need be retained.

Example: If $f(x)=O(x)+O(x \log x)+O\left(x^{2}\right)+O(1)+O(1 / x)$, then

III. When $a$ is a positive constant, then

$$
f(x)=O\left(x^{2}\right) \text {. }
$$

$$
\begin{aligned}
& O(a g(x))=O(g(x)), \\
& o(a g(x))=o(g(x)) .
\end{aligned}
$$

IV. From $f_{1}(x)=O\left(g_{1}(x)\right)$ and $f_{2}(x)=O\left(g_{2}(x)\right)$ it follows that

$$
f_{1}(x) f_{2}^{\prime}(x)=O\left(g_{1}(x) g_{2}(x)\right) \text {. }
$$

Example: $x=O(x), \sin x=O(1)$, therefore $x \sin x=O(x \cdot 1)=O(x)$.

V. From $f(x)=o(g(x)$ it follows that $f(x)=O(g(x))$.

VI. From $f_{1}(x)=O\left(g_{1}(x)\right)$ and $f_{2}(x)=0\left(g_{2}(x)\right)$ it follows that

* Handbuch, p. 139.

$$
f_{1}(x) f_{2}(x)=o\left(g_{1}(x) g_{2}(x)\right) .
$$


whence, substituting $p_{n_{\varepsilon}}$ for $x$ and using (19)

$$
\prod_{k=1}^{n_{z}}\left(1-\frac{1}{p_{k}}\right)=\frac{e^{-C}}{\log \log x+\log (1+o(1))}(1+o(1))
$$

For $\alpha=1$, the expression (5) gives

$$
=\frac{e^{-C}}{\log \log x}(1+o(1)) .^{*}
$$

$$
s_{1}(x)=x \prod_{k=1}^{n} \frac{1-\frac{1}{p_{\lambda_{k}}^{\nu_{k}+1}}}{1-\frac{1}{p_{\lambda_{k}}}}<x \prod_{k=1}^{n} \frac{1}{1-\frac{1}{p_{\lambda_{k}}}} .
$$

Now we obviously have $n \bar{\sum} n_{x}$ and $\lambda_{k} \overline{\overline{ }} k, p_{\lambda_{k}} \overline{\overline{ }} p_{k}$, so that

$$
\prod_{k=1}^{n} \frac{1}{1-\frac{1}{p_{\lambda_{k}}}} \sum \prod_{k=1}^{n} \frac{1}{1-\frac{1}{p_{k}}} \sum \prod_{k=1}^{n_{z}} \frac{1}{1-\frac{1}{p_{k}}}=e^{c} \log \log x \cdot(1+o(1))
$$

according to (20), and (21) then shows that

$$
\limsup _{x=\infty} \frac{s_{1}(x)}{x \log \log x} \overline{<} e^{c} .
$$

A special sequence of integers for which this upper limit is effectively reached is obtained by making

$$
x_{n}=\left(p_{1} p_{2} \cdots p_{n}\right)^{\left[\log p_{n}\right]},
$$

where $\left[\log p_{n}\right]$ is the greatest integer contained in $\log p_{n}$. We then find from (4)

$$
\begin{aligned}
s_{1}\left(x_{n}\right) & =x_{n} \prod_{k=1}^{n} \frac{1-\frac{1}{p_{k}^{\left[\log p_{n}\right]+1}}}{1-\frac{1}{p_{k}}}>x_{n} \prod_{p}\left(1-\frac{1}{p^{\left[\log p_{n}\right]+1}}\right) \prod_{k=1}^{n} \frac{1}{1-\frac{1}{p_{k}}} \\
& =\frac{x_{n}}{\zeta\left(\left[\log p_{n}\right]+1\right)} \prod_{k=1}^{n} \frac{1}{1-\frac{1}{p_{k}}}=\frac{x_{n}}{\zeta\left(\left[\log p_{n}\right]+1\right)} e^{c} \log p_{n}(1+o(1)) .
\end{aligned}
$$

* According to the definition of $o(1)$, we have

$$
\text { whence } \begin{aligned}
& \lim _{x=\infty} \log (1+o(1))=\lim _{\epsilon=0} \log (1+\epsilon)=0, \text { or } \log (1+o(1))=o(1) \\
& \lim _{x=\infty} \frac{\log \log x+\log (1+o(1))}{\log \log x}=1
\end{aligned}
$$

or

$$
\log \log x+\log (1+o(1))=\log \log x \cdot(1+o(1)),
$$

and

$$
\lim _{x \rightarrow \infty} \frac{1+o(1)}{1+o(1)}=\lim _{\delta, \varepsilon=0} \frac{1+\delta}{1+\epsilon}=1 \text { or } \frac{1+o(1)}{1+o(1)}=1+o(1)
$$

whence our formula. 
From (23) we obtain

$\log x_{n}=\left[\log p_{n}\right] \vartheta\left(p_{n}\right)=\left[\log p_{n}\right] p_{n}(1+o(1))=p_{n} \log p_{n}(1+o(1))$, whence

and as

$$
\begin{gathered}
\log \log x_{n}= \\
\log p_{n}+\log \log p_{n}+o(1), \\
\lim _{n=\infty} \frac{\log p_{n}}{\log \log x_{n}}=1,
\end{gathered}
$$

$$
\begin{gathered}
\lim _{n=\infty} \zeta\left(\left[\log p_{n}\right]+1\right)=1,(24) \text { gives } \\
\lim _{n=\infty} \sup _{n} \frac{s_{1}\left(x_{n}\right)}{x_{n} \log \log x_{n}} \overline{>} e^{c} .
\end{gathered}
$$

This relation compared with (22) finally shows that

$$
\limsup _{x=\infty} \frac{s_{1}(x)}{x \log \log x}=e^{c} .
$$

Third case, $0<\alpha<1$.-We begin by developing an asymptotic expression for $\log \prod_{p \leqq x} \frac{1}{1-\frac{1}{p^{\alpha}}}$, where $0<\alpha<1$. As $\pi(n)-\pi(n-1)$ equals 1 or zero according as $n$ is prime or composite, we find by partial summation

$$
\begin{aligned}
\log \prod_{p \leqq x} \frac{1}{1-\frac{1}{p^{a}}} & =-\sum_{p \leqq \infty} \log \left(1-\frac{1}{p^{\alpha}}\right) \\
& =-\sum_{n=2}^{x}(\pi(n)-\pi(n-1)) \log \left(1-\frac{1}{n^{a}}\right) \\
& =\sum_{n=2}^{x} \pi(n)\left(\log \left(1-\frac{1}{(n+1)^{a}}\right)-\log \left(1-\frac{1}{n^{a}}\right)\right) \\
& -\pi(x) \log \left(1-\frac{1}{(x+1)^{a}}\right) .
\end{aligned}
$$

Now we have, by Taylor's theorem,

$$
\log \left(1-\frac{1}{(n+1)^{\alpha}}\right)-\log \left(1-\frac{1}{n^{\alpha}}\right)=\frac{\alpha}{n^{1+\alpha}-n}-\frac{1}{2} \cdot \frac{\alpha}{\left(\xi^{a+1}-\xi\right)^{2}}\left((\alpha+1) \xi^{\alpha}-1\right)
$$

( where $n<\xi<n+1$ )

$$
\begin{aligned}
& =\frac{\alpha}{n^{1+\alpha}}+O\left(\frac{1}{n^{2+\alpha}}\right)+O\left(\frac{1}{\xi^{2+\alpha}}\right) \\
& =\frac{\alpha}{n^{1+\alpha}}+O\left(\cdot \frac{1}{n^{2+\alpha}}\right)
\end{aligned}
$$




$$
\begin{aligned}
\log \left(1-\frac{1}{(x+1)^{a}}\right) & =-\frac{1}{(x+1)^{a}}+o\left(\frac{1}{(x+1)^{2 a}}\right) \\
& =-\frac{1}{x^{a}}+O\left(\frac{1}{x^{1+a}}\right)+O\left(\frac{1}{(x+1)^{2 a}}\right)=-\frac{1}{x^{a}}+O\left(\frac{1}{x^{2 a}}\right),
\end{aligned}
$$

and using (16) we obtain

$$
\begin{aligned}
& \pi(n)\left(\log \left(1-\frac{1}{(n+1)^{a}}\right)-\log \left(1-\frac{1}{n^{a}}\right)\right) \\
& =\left(\frac{n}{\log n}+O\left(\frac{n}{\log ^{2} n}\right)\right)\left(\frac{\alpha}{n^{1+\alpha}}+o\left(\frac{1}{n^{2+\alpha}}\right)\right) \\
& =\frac{\alpha}{n^{\alpha} \log n}+\frac{n}{\log n} o\left(\frac{1}{n^{2+a}}\right)+\frac{\alpha}{n^{1+a}} o\left(\frac{n}{\log ^{2} n}\right)+o\left(\frac{n}{\log ^{2} n}\right) o\left(\frac{1}{n^{2+a}}\right) \\
& =\frac{\alpha}{n^{\alpha} \log n}+O\left(\frac{1}{n^{1+\alpha} \log n}\right)+O\left(\frac{1}{n^{\alpha} \log ^{2} n}\right)+O\left(\frac{1}{n^{1+\alpha} \log ^{2} n}\right) \\
& =\frac{\alpha}{n^{\alpha} \log n}+O\left(\frac{1}{n^{\alpha} \log ^{2} n}\right) \text {, } \\
& \pi(x) \log \left(1-\frac{1}{(x+1)^{a}}\right)=\left(\frac{x}{\log x}+O\left(\frac{x}{\log ^{2} x}\right)\right)\left(-\frac{1}{x^{a}}+O\left(\frac{1}{x^{2 a}}\right)\right) \\
& =-\frac{x^{1-a}}{\log x}+O\left(\frac{x^{1-2 a}}{\log x}\right)+O\left(\frac{x^{1-a}}{\log ^{2} x}\right)+O\left(\frac{x^{1-2 a}}{\log ^{2} x}\right) \\
& =-\frac{x^{1-a}}{\log x}+O\left(\frac{x^{1-\alpha}}{\log ^{2} x}\right) \text {. }
\end{aligned}
$$

Introducing these approximations in (26), we find $\log \prod_{p \leqq x} \frac{1}{1-\frac{1}{p^{\alpha}}}=\alpha \sum_{n=2}^{x} \frac{1}{n^{\alpha} \log n}+O\left(\sum_{n=2}^{x} \frac{1}{n^{\alpha} \log ^{2} n}\right)+\frac{x^{1-\alpha}}{\log x}+O\left(\frac{x^{1-\alpha}}{\log ^{2} x}\right)$. The function $\frac{1}{u^{a} \log u}$ decreasing monotonously when $u$ increases, we have

$$
\begin{aligned}
& \int_{n}^{n+1} \frac{d u}{u^{a} \log u}<\frac{1}{n^{a} \log n}<\int_{n-1}^{n} \frac{d u}{u^{a} \log u}, \\
& \int_{2}^{x+1} \frac{d u}{u \log u}<\sum_{n=2}^{x} \frac{1}{n^{a} \log n}<\int_{1}^{\infty} \frac{d u}{u^{a} \log u}
\end{aligned}
$$

or

$$
\sum_{n=2}^{x} \frac{1}{n^{\alpha} \log n}=\int_{2}^{\infty} \frac{d u}{u^{\alpha} \log u}+O(1)
$$


and in the same way we obtain an approximation for $\sum_{n=2}^{\infty} \frac{1}{n^{\alpha} \log ^{2} n}$, whence $\log \prod_{p \leq x} \frac{1}{1-\frac{1}{p^{a}}}=\alpha \int_{2}^{x} \frac{d u}{u^{a} \log u}+O(1)+O\left(\int_{2}^{x} \frac{d u}{u^{a} \log ^{2} u}\right)+\frac{x^{1-\alpha}}{\log x}+O\left(\frac{x^{1-\alpha}}{\log ^{2} x}\right)$

Integrating by parts, we find

$$
\int_{2}^{x} \frac{d u}{u^{\alpha} \log u}=\frac{x^{1-\alpha}}{(1-\alpha) \log x}-\frac{2^{1-\alpha}}{(1-\alpha) \log 2}+\frac{1}{1-\alpha} \int_{2}^{x} \frac{d u}{u^{\alpha} \log ^{2} u} ;
$$

we also have

$$
\begin{aligned}
\int_{2}^{x} \frac{d u}{u^{\alpha} \log ^{2} u}=\int_{2}^{\sqrt{x}}+\int_{\sqrt{x}}^{x}<\frac{1}{\log ^{2} 2} \int_{2}^{\sqrt{x}} \frac{d u}{u^{\alpha}}+\frac{1}{\log ^{2} \sqrt{x}} \int_{\sqrt{x}}^{x} \frac{d u}{u^{\alpha}} \\
=O\left(x^{\frac{1-a}{2}}\right)+O\left(\frac{x^{1-\alpha}}{\log ^{2} x}\right)=O\left(\frac{x^{1-\alpha}}{\log ^{2} x}\right),
\end{aligned}
$$

and introducing in the expression above, we find

$$
\begin{aligned}
\log \prod_{p \leqq x} \frac{1}{1-\frac{1}{p^{\alpha}}}=\frac{\alpha}{1-\alpha} \frac{x^{1-\alpha}}{\log x}+O\left(\frac{x^{1-\alpha}}{\log ^{2} x}\right) & +O(1) \\
& +O\left(\frac{x^{1-\alpha}}{\log ^{2} x}\right)+\frac{x^{1-\alpha}}{\log x}+O\left(\frac{x^{1-\alpha}}{\log ^{2} x}\right),
\end{aligned}
$$

or finally

(27) $\log \prod_{p \leq x} \frac{1}{1-\frac{1}{p^{\alpha}}}=\frac{1}{1-\alpha} \frac{x^{1-\alpha}}{\log x}+O\left(\frac{x^{1-\alpha}}{\log ^{2} x}\right)=\frac{1}{1-\alpha} \frac{x^{1-\alpha}}{\log x}(1+o(1))$

On account of $n \bar{\Sigma} n_{x}$ and $p_{\lambda_{k}} \overline{\overline{ }} p_{k}$, equation (5) gives

$$
s_{a}(x)<x^{a} \prod_{k=1}^{n} \frac{1}{1-\frac{1}{p_{\lambda_{k}}^{a}}} \overline{<} x^{a} \prod_{k=1}^{n} \frac{1}{1-\frac{1}{p_{k}^{a}}} .
$$

Substituting $p_{n_{z}}$ for $x$ in (27), we obtain by the aid of (19), manipulating the symbol $o(1)$ in the same way as when deriving (20) from the formula preceding it,

whence, by (28)

$$
\log \prod_{k=1}^{n} \frac{1}{1-\frac{1}{p_{k}^{a}}}=\frac{1}{1-\alpha} \frac{(\log x)^{1-\alpha}}{\log \log x}(1+o(1)),
$$

$$
\limsup _{x=\infty} \frac{\log \frac{s_{a}(x)}{x^{\alpha}}}{\frac{(\log x)^{1-\alpha}}{\log \log x}}=\frac{1}{1-\alpha}
$$


To obtain a special sequence of integers for which this upper limit is effectively attained, make

We then obtain from (5)

$$
x_{n}=p_{1} p_{2} \cdots p_{n}
$$

$$
s_{a}\left(x_{n}\right)=x_{n}^{a} \prod_{k=1}^{n} \frac{1-\frac{1}{p_{k}^{2 a}}}{1-\frac{1}{p_{k}^{a}}}
$$

and we have

$$
\begin{aligned}
\log \prod_{k=1}^{n}\left(1-\frac{1}{p_{k}^{2 a}}\right) & =-\frac{1}{1-2 \alpha} \frac{\left(\log x_{n}\right)^{1-2 a}}{\log \log x_{n}}(1+o(1)) \text { for } 0<\alpha<\frac{1}{2} \text { (by (29)), } \\
& =-\log \log \log x_{n}+O(1) \text { for } \alpha=\frac{1}{2} \text { (by (20)), } \\
& =O(1) \text { for } \frac{1}{2}<\alpha<1 \text { (infinite product convergent), }
\end{aligned}
$$

so that in all three cases

$$
\log \prod_{k=1}^{n}\left(1-\frac{1}{p^{2 a}}\right)=\frac{\left(\log x_{n}\right)^{1-a}}{\log \log x_{n}} \cdot o(1) .
$$

Equations (31) and (29) then give

or

$$
\log \frac{s_{a}\left(x_{n}\right)}{x_{n}^{a}}=\frac{1}{1-\alpha} \frac{\left(\log x_{n}\right)^{1-a}}{\log \log x_{n}}(1+o(1))
$$

$$
\lim _{n=\infty} \frac{\log \frac{s_{a}\left(x_{n}\right)}{x_{n}^{\alpha}}}{\frac{\left(\log x_{n}\right)^{1-\alpha}}{\log \log x_{n}}}=\frac{1}{1-\alpha} .
$$

and we finally obtain, by combining (30) and (32)

$$
\limsup _{x=\infty} \frac{\log \frac{s_{a}(x)}{x^{\alpha}}}{\frac{(\log x)^{1-\alpha}}{\log \log x}}=\frac{1}{1-\alpha} \quad(0<\alpha<1) .
$$

